\title{
The Mirror Image and Narrative Meaning of "Saturday" in Michael Eun's Works
}

\author{
Jing Li \\ Luohe Medical College, Luohe, Henan, 462000, China
}

\begin{abstract}
"Mirrors" "shutters" and other objects with function of reflection, mapping, or self-mapping through perceptual reality to constitute a series of mirror image combinations that run through Ian Mc Ewan's novel "Saturday", it has assumed the important narrative function and it has the very profound meaning. Mirror image combination not only assumed the important narrative function, but also metaphor the gaze of the gaze on the subjectivity, which reflects the indifference and alienation between people.
\end{abstract}

Keywords: microscope, shutter, rearview mirror, mirror image, gaze, narrative function

\section{Introduction}

Mirror is not only finishing the makeup of the objects, it is the human spirit and cultural connotation of the important material carrier. Mirror is not only in the daily life, but also into the human life in all the key areas, such as birth, marriage, funeral, divination, sacrifice and so have the appearance of mirror, formed a rich mirror culture. At the same time, the image of the mirror penetrated into the field of literature, into the myths, legends, novels, poetry narrative. "Mirror", "rearview mirror" and other objects in the shape similar to the mirror, with a reflection or mapping function, that is, with the function and role of the mirror is the "mirror" function of the extension And extensions. "Shutter" is the reality of the mirror image of the carrier, can be self-mapping through the perception of reality. This series of mirrored images gives the protagonist Bellanan a view of 
the acquisition of a person's perception, truth and power, and strengthen the view as a dominant discourse and hierarchical order to build one of the ways.

\section{The medical gaze under the "microscopes"}

In modern medicine, "vision is scientific and it not only see while distinguishing features, identify differences, make judgments." In traditional medicine, doctors solve the problem by visually and auscultating the patient's body. Modern medical manipulation of microscopes, magnifying glass, scanners and other medical technology, the gaze will penetrate into the body, to detect hidden within the body and constitute a visible image, any disease in the stare and medical language can be resolved. People in the visual power under the control of completely exposed, power through the medical gaze and discourse spread to the individual body. "

Without intervention, the visual and analytical visualization makes the truth appear and the truth manifests itself. In the novel Saturday, Berroan is portrayed as a typical gaze. As a brain surgeon, Beloan has always been very good and very sharp "vision." Berroan's "judgment at the first glance" of the brain disease, which ensures that he is extremely effective and accurate in obtaining "truth" and truth through "sight"; secondly, with intensive surgery, Success rate, as he established a medical master --- the truth and authority --- the status of medicine. "The surgery will not make him feel tired - once he is immersed in the hospital, the operating room and well-organized surgical procedures constitute a closed world, concentrate on the surgery from the

Microscopic prying vivid path until reaching the lesion, whenever this time he burst out of superhuman ability, more like a desire, the extreme desire to work. "In a sense," microscope "reduced to the main body of self-order and discourse power construction tool, metaphor through the visual search for truth and truth desire machine.

It is worth noting that Bei Luoan subjectivity is constrained by the carrying of knowledge-power coding of the medical gaze, subjectivity can not be extended to the elusive, with the uncertainty of the Although the enthusiasm for the operation is high, but for Friday afternoon's desk work - referral applications and the reply to the application, prepare summary of the meeting, to be completed at the same time to assess, on the work of the field. Planning management recommendations -- quite tired. General silence in medical gaze and self-speech Language "and" hard-han style "under the situation of the difficulty and risk doubling of the operation; the mental and physical exhaustion and the speech retardation of" reply "," summary "," evaluation "and" suggestion " Berroen stays in medical gaze for a long time, but avoids the communication and communication between patients and doctors, doctors and supervisors, doctors and co-workers, the subject appears in anxiety and anxiety in the field of interpersonal communication, and subjectivity is extremely distorted. For him, as well as knowing the patient, knowing the whole situation of society and others without having to communicate by words, it is more than enough to gaze with medicine alone, and 
therefore Beiluoan, through medical gaze, seeks truth and truth, builds his superior hierarchical order and has the right to speak, oppression of the marginal group of other words, the relationship between people become equal to the opposition, indifference and alienation, for the modern society inevitably interpersonal conflict foreshadowed.

\section{The panoramic gaze outside the "shutters"}

"Impersonal Panoramic Open Gaze is an effective tool for disciplining the society, is the 'power of the eye', it will become his own watchdog, the power to achieve the automatic operation of colleges and universities." Foucault's stare broad To our lives, not only limited to schools, hospitals, but also extends to the use of transparent glass and non-partition space arrangement, but also applies to the novel in the recurrent shutters. "Shutters" will be full of warmth and comfort of the indoor and London Square connected, symbolizing the superiority of Beiluo and social status. Staring at the move by Bei Luoan described as "moments of beauty." The window as a mapping of the outside world objects, with metaphorical significance. The window is the hero's home, "is to give the ownership and security of space." Outside the window of the world connected to the desire for gaze, as a window of internal and external relations directly reflects his situation. The hero says, "The city is a great achievement, brilliant creation and natural masterpiece", and his own home is the eighteenth century dream of bathed in the light of modern civilization. "However, the window space is relatively closed and isolated private areas, set the power and technology of the" layers of preparedness ", such as" Ham door lock "," the door to the door " "The door alarm", "door chain", "door mirror", "electronic anti-theft device", "emergency alarm button"; window outside the world is the distribution of some social uncertainties, such as beggars, drug addicts and hooligans. As a window of internal and external relations, in the affiliation and security at the same time, become a subject of captive space. Staring at the constructed ideal world is just a harmonious, poetic appearance, obscuring some kind of real existence, with the imprint of power. Staring at the object is no shortage of drug addicts in the dispute couple, there are more than two hours hovering boy, holding the rails crying young man, but also a whiskey from the hand of the crazy woman. When the depth of gaze approaching madness, Bei Luoan had imagined "himself is Saddam, is standing on a balcony in the Baghdad government building, contentedly review the following people." From the point of view, panoramic gaze has become a "medical gaze" extension and expansion ", watching or in a high position on the ground or hold the right to speak, constantly looking at being seen by the beholder or on their own situation without the knowledge or longterm loss of the right to speak." 


\section{The "window" and "rear-view mirror" image}

"Transparent space" in the "window" symbolizes Beiluo An noble temperament and superior identity, "rearview mirror" repeatedly appeared in the car scratch narrative, threatening the noble qualities and superior identity, but also The protagonist's staring action is constructive and heralds the tensions between the characters. "Car window" to build the car space can also allow the subject to achieve visual reality, self-mapping. Therefore, the window can be counted as a mirror image and it is a transparent space with the symbol of the superiority of Berroan identity and noble temperament. Beiluo An ashamed to admit that more like in the luxury car closed space to watch the city, enjoy the clean air and the "Schubert Trio", suddenly feel the narrow streets also look noble. "Narrow streets become noble up" What is the solution? Not so much the streets become noble, it is better that Berro in the luxury car space --- excellent and comfortable environment and elegant music --- build their own noble identity, so its staring at the car space is at best their own noble Empathy and Strengthening of Temperament. "Mirror" repeatedly appeared in the car scratch narrative, threatening the noble qualities of Berroan and superior identity. "Mirrors" threaten the superior subjectivity of Bellan's construction, and the three small bums reflected in the rearview mirror are external threats to superior subjectivity. Therefore, after some hesitation and ideological struggle, Berroan out of the car in the contradictory mood, even more at a loss. Ambivalence means that the subject is unbalanced in the self-constructed noble temperament and the power discourse. The protagonist sought evidence in his favour, but when he realized that the rear-view mirror was intact, the street was empty, and the parade could not get involved,

"Mirror" on the protagonist of the gaze of action also has a constructive role. Behroan's right hand has been put on the door handle, but he suddenly stopped the action, choose to wait and see from the rear-view mirror, this purpose is to wait and see is a staring strategy to help understand the other person's power distribution As well as status. From the rear-view mirror can be perceived three shadows, patronize dancing dance club to make their identity become elusive, so that he is difficult to cope with; the hero through the other BMW models speculate that they

Social identity, it is difficult to criminal or drug transactions with them in a piece. Obviously, the rearview mirror has a mapping function, is the reflection of the reality of the object, and Behroan's gaze imprinted in his value judgments on the other in the psychological anxiety, but also a necessary means of continuing medical gaze. In the car to stare at the other person's strange footwork, behavior posture, wearing costumes, the viewer attempts to obtain valuable discourse information, enhance their authority and control of discourse, but the "hands of that guy

But with the rearview mirror, presumably to be used as evidence "Obviously full of anxiety and unease." Both sides of the contradictions intensified in the issue of rear-view mirror compensation, the rearview mirror is also a symbol of the right to speak for contention. In the car rearview mirror and Baxter rupture of 
the rearview mirror, the hero can be observed Baxter "shaking hands", even in the face of beating after his subconscious also left a doctor's diagnosis, in medical terms " Gamma-aminobutyric acid is too low, neuronal cell problems "and" two enzyme deficiency "to find the root of moral and ethical. Therefore, the rear-view mirror is the novel contradiction and conflict, and the gaze of the protagonist The role of the right to speak is constructive with this image, Bei Luoan will medical gaze function from the "cognitive function" level to "language function" and "power function" level - Beiluo An identification of disease through dialectical The discourse of knowledge, the power to inquire, the medical stare and medical discourse power, the passive situation as the victim, the space of power is redivided into the medical field.

\section{Conclusion}

Berean made a medical gaze at Baxter in an attempt to escape a beaten, not sincerely "concerned" about Baxter, but under the guise of hypocrisy charity, to exercise deception and disciplinary reality on the lower classes. Baxter's backlash and subsequent retaliation also demonstrated the resistance of the gazer to the surveillance barrier. Such gaze and anti-stare instead of digestion but exacerbated the tension between the two sides. We can glimpse how gaze works from a series of mirror image combinations, such as the microscope, the shutter, the rear-view mirror and so on. Mirror image combination not only assumed the important narrative function, but also metaphor the gaze of the gaze on subjectivity, which reflects the indifference and alienation between people in contemporary British society.

\section{References}

[1] Mao Lingying: "The Scarlet Letter in the mirror image and its narrative meaning", "foreign literature", 55(12), pp.68-70, 2011

[2] Chen Yung, Zhao Yifan, et al: "Gaze", "Key Words of Western Literary Theory", Foreign Language Teaching and Research Press, pp.87- 91, 2006

[3] Michel Foucault, Yan Feng, "The Eye of Power: An Interview with Foucault," Shanghai People's Publishing House, pp. 18- 20, 1997

[4] Zhu Xiaolan: "Gaze "theoretical research", Nanjing University master's thesis, pp.58- 61,2011

[5] Ian McEwan, Xia Xin Zhuo: "Saturday", Shanghai Translation Publishing House, pp.85-87, 2014 